\title{
Acerca de la justicia interétnica en perspectiva dela cultura jurisdiccional. Los trayectos entre Jujuy y la Audiencia de Charcas, siglo XVI
}

\author{
About interethnical justice in the perspective of the jurisdictional culture. \\ XVI century paths between Jujuy province and Charcas Audience \\ María Cecilia Oyarzabal \\ Universidad Nacional de la Patagonia-Argentina \\ mariac.oyarzabal@gmail.com
}

\section{Resumen}

La construcción de la figura jurídica del indígena, aquel vasallo libre pero rustici, miserabiles personae y perpetuo menor de edad, necesitado de protección, resultará cardinal en la conformación de la sociedad colonial. Su status diferenciado y las prerrogativas que de él se desprenden configurarían un particular uso de la justicia a través de las diversas esferas: las autoridades étnicas, el Cabildo, la Gobernación y la Real Audiencia. En las últimas décadas, la Nueva Historia del Derecho proveyó las herramientas teóricas para repensar las relaciones de poder y la potestad de establecer derecho del período en clave de cultura jurisdiccional. El análisis de expedientes extraídos del Archivo de Tribunales de San Salvador de Jujuy y el Archivo y Bibliotecas Nacionales de Bolivia nos permitirá repensar los vínculos existentes entre las diversas instancias de justicia y las posibilidades que esos procesos pudieron suponer para las poblaciones indígenas del Jujuy colonial en el marco de pertenencia a la Real Audiencia de Charcas.

Palabras clave: indígenas, justicia, Real Audiencia, Jujuy.

\begin{abstract}
The construction of the legal entity of indigenous, a free vassal but rustici, miserabiles personae, perpetual child in need of protection, will result on a central concept in the shaping of the colonial society. His distinct status and prerogatives that flow from him shaped a particular use of justice across various spheres: ethnical authorities, the Cabildo, the Government and the Royal Audience. On the last decades, the New Law History provided theoretical tools to rethink power relationships and the authority for making justice during this period, based on jurisdictional culture. The analysis of case files from archives of San Salvador de Jujuy Court, the National Archive of Bolivia and from National Libraries of Bolivia will allowed us to rethink about the connections between different instances of justice and which possibilities it implies for the native populations of colonial Jujuy in the context of its membership to the Royal Audience of Charcas.
\end{abstract}

Key words: indigenous, justice, Royal Audience, Jujuy

Autoctonía. Revista de Ciencias Sociales e Historia Vol. IV, Nº2, Julio-Diciembre 2020, 181-193 ISSN 0719-8213 


\section{Introducción}

La conformación de la sociedad colonial contó con recursos de valía para consolidar su hegemonía entre la población conquistada y legitimar su dominación. En el entramado de poder que discurrió entre las ciudades, los pueblos y la campaña, se generó un interjuego de tensiones, intereses y redes del que los indígenas no fueron ajenos. Por el contrario, en diferentes contextos, su figura - construida a partir de su particular status jurídico- tendrá un rol de sumo interés en el mismo.

En esencia, la concepción jurídica del indígena actuó como un elemento constitutivo en la afirmación ideológica del dominio castellano, reduciendo discursivamente el signo opresivo de la empresa de dominación a través de la idea de protección a los naturales del continente. Así, aquel vasallo libre pero rústico, miserable y perpetuo menor de edad, tendría un lugar específico en este entramado de poder a través de las posibilidades que le brindaba, en la justicia, la especificidad de estas antiguas categorías resignificadas en el contexto americano. Su ignorancia de la cultura letrada y su carácter de neófitos en la fe constituían la base de su rusticidad, característica que les daba la prerrogativa de apelar a la costumbre en lo concerniente a asuntos particulares (Hespanha, 1993; Clavero, 1994). Asimismo, eran especiales objetos de caritas, a partir de la estimación de que carecían de los recursos necesarios para desenvolverse socialmente; es decir, eran personae miserabilis (Duve, 2005; Agüero, 2012). La sumatoria de estas condiciones igualaba a los indígenas con los menores de edad y asumía el imperativo de que fuesen tutelados. De esta manera, la libertad quedaba limitada por el atributo de protección "conformándose así un particular estatus étnico que implicaba una subjetividad disminuida, sometida a la tutela perpetua del rey y de la Iglesia" (Agüero, 2012: 249).

El estudio de los sistemas jurídicos se constituyó, tradicionalmente, como el campo de investigación de la Historia del Derecho. Desde los clásicos y debatidos estudios de derecho indiano que negaban la condición colonial de América y reducían el rol de los indígenas a su status nominal de vasallos (Levene, 1951), la disciplina se preocupó por variables tales como los contextos culturales e históricos que posibilitaron la aparición de esos sistemas, siempre contemplados dentro del marco institucional (Levaggi, 2012). En las últimas décadas, la interpretación del orden jurídico del mundo americano se ha profundizado a través de variables propias como el casuismo (Tau Anzoátegui, 1992), el derecho local (Duve, 2005; Tau Anzoátegui y Agüero, 2013) o la localización del derecho (Agüero, 2013), revisando la capacidad de agencia de los actores sociales en el contexto colonial. Sin embargo, consideramos que la transformación más profunda en este campo se llevó a cabo a través de la reinterpretación de la idea de poder en las sociedades de Antiguo Régimen. 
Desde Portugal, Antonio Hespanha (1989; 1993) brindará un aporte basal, a partir de descartar los enfoques individualistas propios de la teoría social dieciochesca, para volver la mirada a la concepción medieval que contemplaba la existencia de un orden universal donde se concatenaban los diversos cuerpos sociales, asumiendo funciones diferenciadas. Esta evidencia suponía que la forma de gobierno imperante debía ser congruente con este ordenamiento: "reposar en la autonomía político-jurídica de los cuerpos sociales y respetar su articulación natural” (Hespanha, 1989: 235). Entre otros aspectos, esta mirada desestimó la presencia de mecanismos de representación institucional que involucraran la participación de la sociedad toda en la vida política (Hespanha, 2002). Un cuerpo social constituido en estos términos no admite un poder central que lo discipline; es así como se arriba a la conclusión primordial propuesta por este modelo: la ausencia de Estado en la Europa de Antiguo Régimen.

En su lugar, será la potestad de decir derecho la que establezca el poder político, génesis de la cultura jurisdiccional (Costa, 1969). La Iurisdictio constituía una potestad pública, de acuerdo a la lógica corporativa de la sociedad por la cual sus titulares eran detentores de la facultad de establecer normas y dirimir pleitos. Entre ellos, el Cabildo, como núcleo político de la ciudad, compone un sujeto corporativo con facultades de gobierno para decidir sobre los destinos políticos y económicos de todo el territorio que queda bajo su jurisdicción. Esta mirada trasladada al contexto americano permite abrir la perspectiva a la relativa autonomía en la toma de decisiones que frecuentemente se habían explicado en clave de distancia entre aquellas sociedades periféricas y el gobierno central (Agüero, 2008). El modelo jurisdiccional nos brinda un cuadro de autoridades de doble vertiente: el poder regio que se extiende en forma descendente a través de virreyes, audiencias, gobernadores, corregidores y visitadores, y el poder del Cabildo, núcleo político de la ciudad, que constituye un sujeto corporativo (Agüero, 2007; 2008).

Los pueblos de indios, si bien se comprenden dentro de la jurisdicción de la ciudad, dependerán de las decisiones tomadas a nivel de la Real Audiencia, el Corregimiento o la Gobernación. Internamente, el Cacique será reconocido como el señor natural de la población indígena y ocupará un rol determinante en la estructura del orden colonial, detentando atributos menores para impartir justicia. Su desempeño estará acompañado por el Alcalde indígena, figura introducida en el marco del modelo impuesto por el virrey Francisco de Toledo de la república de indios y la república de españoles. Si bien, a partir de este ordenamiento, los pueblos de indios cuentan con sus propios cabildos, a estos se les negará la cesión de la potestad para decir derecho. Es por ello que, necesariamente, la justicia del medio indígena estará subsumida a la justicia de la ciudad española. Como nexo entre estas dos esferas, se creará la figura del Protector de Naturales, una autoridad que debía intervenir en cualquier caso en donde hubiese un indígena implicado, expresión del vínculo paternalista que la Corona asumía para con estos vasallos perpetuos menores de edad y objeto de caritas (Agüero, 2012). Este mapa jurisdiccional se completará; cobrará un sentido renovado en la medida en que los actores sociales utilicen 
indistintamente unas y otras instancias, sopesen sus posibilidades, se muevan en el espacio. Entre ellos, los indígenas harán uso de las alternativas que se les presentan.

Hablamos de "justicia interétnica" y con ello nos referimos a "una práctica mediada por la preeminencia de una institución de control social signada por la desigualdad, basada en la diferencia étnica" (Oyarzábal, 2019: 80). Consideramos que el acceso a la justicia por parte de los indígenas siempre será en condiciones de inferioridad por estar insertos dentro de la lógica de dominio colonial y la violencia que la misma entraña. $\mathrm{Al}$ hacerlo, además, el indígena refrenda el vínculo colonial porque, implícitamente, lo admite (Poloni Simard, 2005). A pesar de estas aclaraciones, resulta difícil comprender la sociedad estudiada sin considerar la relevancia que, en determinados contextos, adquiría la relación entre indígenas y justicia. Consideramos que el modelo jurisdiccional permite abrir la mirada sobre el vínculo que los indígenas podían forjar con la justicia. A partir del balance/tensión de poderes entre la Gobernación, la Audiencia, el Cabildo y las autoridades étnicas, se abren nuevas posibilidades de estrategias que exploraremos tomando como referencia tres casos que nos resultan ilustrativos. El objetivo es pensar las variables que juegan dentro de la relación entre las diversas esferas de acuerdo a la lógica de la cultura jurídica imperante y las posibilidades que los indígenas encuentran y esgrimen en ese contexto.

\section{Indígenas andando las jurisdicciones}

La segunda mitad del siglo XVI significó, para la frontera sur del novel Virreinato del Perú, un tiempo de expansión. Clausurado el período de las guerras civiles, en 1559 se instituye la Audiencia de Charcas y se consolida un ciclo de fundaciones que había inaugurado seis años antes la ciudad de Santiago del Estero, en el territorio que quedaría bajo la Gobernación del Tucumán, Juríes y Diaguitas, establecida en 1563. Ese vasto espacio político correspondía a la jurisdicción de la Audiencia de Charcas con sede en la ciudad de La Plata, Audiencia Subordinada de menor jerarquía en lo formal que la Audiencia Virreinal que para América del Sur correspondía a Lima (Tau Anzoátegui y Martiré, 1996).

Este ciclo de fundaciones se cerraría, en 1593, con la colocación del rollo de justicia en el espacio que, de ahí en más, sería la plaza de San Salvador de Jujuy. La región, estrechamente vinculada a las zonas andinas centrales, se extendía a través de una geografía signada por un amplio corredor trazado por el Río Grande y se repartía por diversos biomas que iban desde la aridez de las alturas de la Puna hasta las selvas subtropicales del piedemonte andino (Salas, 1945; Sica, 2006). Las mercedes iniciales fueron otorgadas en la primera mitad del siglo XVI desde Charcas (Sica, 2006), aunque una tenaz resistencia indígena liderada por el cacique Diego Viltipoco obstaculizaría las posibilidades de ocupación efectiva del espacio. Recién en el año 1595, el líder rebelde fue capturado junto a algunos de sus capitanes y, con ello, se neutralizó la amenaza que suponían los grupos que respondían a su liderazgo, según consta en la Probanza de Méritos y Servicios de Francisco de Argañaraz, el fundador de la ciudad (Levillier, 
1918-1919). La historia de rebelión directa por parte de Viltipoco, sin embargo, se alterna con episodios de negociación y reconocimiento hacia las autoridades castellanas. La crónica de uno de estos episodios constituye nuestro primer caso de análisis.

\section{Diego Viltipoco, la Real Audiencia y el Gobernador}

Cerca de 1588, Fray Reginaldo de Lizárraga, el Provincial Dominico en los territorios de Chile y Río de la Plata, transita la geografía que años más tarde correspondería a la jurisdicción de Jujuy. En el extenso recorrido con el que procura reconocer las regiones sobre los que ejerce su autoridad, Lizárraga ([1605], 1999) escribe una extensa crónica que titularía "Descripción del Perú, Tucumán, Río de la Plata y Chile”, en la que deja una sucinta pero por demás interesante descripción del accionar de Diego Viltipoco. Según su relato, Viltipoco, como Cacique principal de la región, había enviado una comitiva a la Real Audiencia a fin de negociar su cooperación con los conquistadores, poniendo como requisito no quedar bajo la autoridad de ningún encomendero sino directamente del Rey. A cambio, el Cacique prometía pagar un tributo moderado, ocuparse de los tambos de la región, proveer de mano de obra a la explotación del Cerro de Potosí y admitir la entrada de sacerdotes que evangelizasen a los indígenas de su pueblo (Lizárraga [1605] 1999: 353).

Una vez arribado a Salta, Lizárraga recoge las novedades surgidas en torno a las acciones de Viltipoco. Juan Ramírez de Velasco, gobernador del Tucumán al enterarse del acuerdo alcanzado entre el Cacique rebelde y la Corona, había enviado un grupo de soldados para tomar posesión de las tierras que quedaban bajo el dominio de Viltipoco y efectivizar su incorporación a la provincia. La respuesta del Cacique había sido contundente; en palabras de Lizárraga, "les dijo se volviesen a Tucumán, donde habían salido, porque no había de ser sujeto a aquella gobernación sino a la Audiencia de los Charcas; donde no, los haría matar a todos" (Lizárraga [1605] 1999: 353-354). En este período se advertía, aún, una débil presencia castellana en la Gobernación del Tucumán. Una de las razones de ello era, justamente, la resistencia de Viltipoco, que bloqueaba el paso entre aquella región y las zonas centrales.

El contexto de violencia propio del período inicial favoreció una decisión como la que tomó el Cacique, quien no desestimó, sin embargo, el vínculo de autoridad existente en la jurisdicción y dentro del cual se movía. Las Reales Audiencias constituían la representación directa del Rey en cada distrito audiencialy sus decisiones contaban con legitimidad real. Además de estar por encima de la autoridad de los gobernadores, su función principal residía en reparar las arbitrariedades llevadas a cabo en las instancias inferiores (Garriga, 2010; Barriera, 2013; Angeli, 2016). En su respuesta, Viltipoco reconoce la autoridad de esta sobre el Gobernador y desdeña la pretendida imposición de Ramírez de Velasco. La construcción del vínculo entre el Rey y sus vasallos requería demostraciones de benevolencia cuasi paternal por parte del soberano. La flamante sujeción de los indígenas, en la que la idea de protección estaba tan presente, hacía más profunda aún esta necesidad. La Audiencia, como representante del Rey en estos territorios, 
recogía esta función de benevolencia para con sus súbditos más desprotegidos. Si bien no era este el caso de Viltipoco, que se había mostrado remiso a entrar en policía cristiana, reconocemos que existe una proyección de esto en los privilegios observados para con los indígenas en general: "tienen caso de Corte" (Solórzano Pereira, [1648] 1703: 234); es decir, ostentan la prerrogativa de asistir a la Real Audiencia directamente ante un conflicto pasando por sobre la potestad jurisdiccional del Cabildo. De este privilegio hará uso un cacique del pueblo de Humahuaca, como veremos a continuación.

\section{Pedro Socomba, la Real Audiencia y el Cabildo}

El pueblo de indios de Humahuaca se estableció en 1595 en la zona septentrional de la quebrada homónima, una región densamente poblada con una identidad cultural propia. Su estratégica ubicación en las rutas comerciales que unían la Puna y el centro del Virreinato con sus dominios meridionales, además de condiciones ecológicas propicias para el cultivo y la cría de ganado, hicieron de San Antonio de Humahuaca el más importante de los pueblos de indios de la jurisdicción (Salas, 1945; Sica, 2006; Zanolli, 2008; Nielsen, 2010). En 1641, don Pedro Socomba, Cacique principal del pueblo, presenta un requerimiento, a través del Protector de Naturales, ante la Audiencia de La Plata, sita a más de 600 kilómetros de Humahuaca. La finalidad de la presentación estriba en reducir el número de mitayos que el pueblo de Humahuaca debía enviar a la ciudad de San Salvador de Jujuy. El sistema de mita, implementado en la segunda mitad del siglo XVI por el virrey Francisco de Toledo, se basaba en la forma de trabajo rotativo propia del mundo incaico que se resignificaría como una imposición del orden colonial. Mita de plaza se denominó a las prestaciones de mano de obra exigidas a fin de mantener y ampliar las obras públicas. En la Gobernación del Tucumán, donde las ciudades no habían experimentado la bonanza de otros espacios del Virreinato, el aporte de la mita de plaza sería por demás significativo (Sica, 2014b). Para las comunidades, sin embargo, el envío de un grupo de indígenas a realizar obras a la ciudad constituía una importante carga. Si este fue el caso de la parcialidad del cacique Socomba no lo sabemos específicamente, ya que el camino elegido para presentar su reclamo ante el máximo tribunal de la jurisdicción tomó otro derrotero. El argumento que justifica su pedido se basa en que la iglesia del pueblo "esta cayéndose"1, por lo que era preciso que seis de los mitayos que debían ser enviados a la ciudad se ocuparan en la tarea de reedificar el tempo.

La demanda es atendida y, esta vez, el propio Socomba viaja a San Salvador de Jujuy con la documentación que da cuenta de ello. Los primeros días del año siguiente lo encuentran haciendo su presentación ante el Cabildo y el gobernador Miguel de Sesé, según consta en actas:

“pareció don Pedro Socomba indio cacique principal del pueblo de Omaguaca de esta jurisdicción y presentó esta real provisión de la Real Audiencia de la Plata y pidió su cumplimiento y habiéndose fecho relación de ella por mí el presente escribano su señoría de dicho señor gobernador le tomo en sus manos beso y dijo que obedecía" 2 . 
La acción de tomar el documento y besarlo en signo de reverencia a la firma que sustituye la mano del Rey no era más que parte del ritual estipulado para estos casos. Sin embargo, la mirada del portador de la resolución resignificará este gesto reglado. En máximo tribunal de la jurisdicción, la representación del Rey en ella hace lugar al pedido de un indígena y su materialización en papel es objeto de reverencia, no solo por parte de los miembros del Cabildo sino del propio gobernador, subvirtiendo las tradicionales relaciones de sujeción dentro de la jurisdicción. Ciertamente, don Pedro Socomba no es un indio del común. Su nombre se cuenta entre los fundadores de la cofradía de la Santísima Virgen de Copacabana junto al Obispo del Tucumán, el cura del pueblo y su encomendero, don Pedro Ochoa de Zárate. La cofradía era beneficiaria de una sementera que producía papa y trigo destinada tanto al mercado local como a los asientos mineros jujeños o el propio Potosí. Socomba, junto al sacerdote y el mayordomo de la cofradía, sería el responsable de todos estos movimientos económicos (Zanolli, 2005; 2008). Además de su rol como Cacique principal de un pueblo de las características de Humahuaca, estos rasgos de su historia dan cuenta de que pertenece a ese sector de la élite indígena que pudo hacerse un lugar adecuado dentro de la estructura de poder colonial.

Cuando estudiamos la justicia interétnica nos atraviesa el interrogante de cómo la justicia impuesta llega al indígena, en qué medida estos se apropian de ella como herramienta, a través de qué vías llegan a un tribunal. La historia de Socomba en la fundación de la cofradía que se había dado en 1634 nos habla de un acercamiento al aparato cultural impuesto, un manejo de las herramientas provistas por el invasor. Resulta interesante también ver que el primer viaje a la Audiencia lo realiza el Protector de Naturales en nombre del Cacique, pero será Socomba en persona quien lleve al Cabildo el dictamen que lo favorece. El viaje de don Pedro Socomba compone un acto político en el cual reivindica sus derechos como indígena hacia el poder español y refrenda su rol de autoridad hacia el interior de la comunidad. Es también una acción profundamente legitimante del orden colonial, su capacidad de agencia en tanto autoridad de la comunidad está totalmente permeada por la cultura jurídica impuesta. Socomba admite poner a disposición mano de obra, solo plantea la necesidad de redireccionarla. Esa dirección, justamente, será la construcción de la iglesia, expresión material de las políticas evangelizadoras en el pueblo de indios. Su rol como Cacique le permitió esta función articuladora hacia dentro, hacia su propia comunidad, y hacia el afuera: la ciudad de españoles y los ámbitos de justicia que el sistema pone a su disposición. Serán estos privilegios propios del status jurídico de los indígenas los que les otorguen cierto margen de maniobra dentro del mundo colonial. Este parece ser el caso de Francisco Vilca, con el que cerramos el presente estudio.

\section{Francisco Vilca, la Real Audiencia y el Alcalde indígena}

Los recursos minerales constituyeron algunas de las riquezas que Jujuy aportó a la Corona de Castilla, muy especialmente a través de las explotaciones auríferas y argentíferas de las Puna (Sica, 2006; Albeck y Palomeque, 2009; Becerray 
Estruch, 2011; Becerra, 2012; 2014). Inicialmente sujeta al Cabildo jujeño, la Puna contó, desde 1595, con una jurisdicción propia a partir de la creación de la figura del Corregidor para el valle de Omaguaca que, en la segunda década del siglo XVII, se convertiría en Teniente de Gobernador y Justicia Mayor, cargos que asumirían funciones de justicia que incluían las referidas a la minería (Sica, 2006; 2014; Albeck y Palomeque, 2009; Estruch, 2009; 2012; Becerra y Estruch, 2011; Becerra, 2014). Tal creación dio lugar a una serie de conflictos jurisdiccionales que han sido estudiados convenientemente por Dolores Estruch y Florencia Becerra (2011). Profundizando en un caso también abordado por las autoras, nos interesa subrayar la agencia indígena en este entramado pleno de tensiones que supone la explotación minera en un contexto de jurisdicciones múltiples.

En 1657 se presentan ante la Real Audiencia de Charcas el presbítero Esteban Gajardo y el escribano de Cámara de la Real Audiencia, Juan de Cabrera Girón. Ambos se adjudican ser los beneficiarios de la mina de Las Ánimas situada en el Valle Rico, en la Puna jujeña ${ }^{3}$. En lo que refiere a la adjudicación de explotaciones de mineral, las Ordenanzas del Virrey Toledo estipulaban que sería beneficiario de una veta quien primero la encontrase y la manifestase ante la justicia (Lorente, [1573] 1867). Siguiendo con lo sugerido por Juan de Matienzo en su obra Gobierno del Perú (Lohmann Villena, [1567] 1967), el virrey estableció que tendría prioridad quien registrase en primer lugar, acción que indefectiblemente debía llevarse a cabo dentro de los treinta días subsiguientes al hallazgo.
De acuerdo a su declaración, Esteban Gajardo había descubierto la veta de mineral y decidido hacer la manifestación en la ciudad de Potosí, documento que se asienta el 10 de febrero de 1657. Entretanto, un indio que respondía a Cabrera Girón, Francisco Vilca, habría robado metal de esa misma veta para presentarlo ante el Alcalde indígena Alonso Cusi, aseverando ser su descubridor ${ }^{4}$ (Becerra y Estruch, 2011; Becerra, 2014). La declaración de Francisco Vilca, previsiblemente, expondrá otra versión de los hechos. Según su testimonio, exploraba la zona cateando, por orden de Cabrera Girón, cuando halló la veta de oro que prontamente manifestó ante el mencionado Alcalde indígena, Alonso Cusi. Esta presentación realizada en la instancia de justicia más próxima a la veta y privativa de la población indígena se realiza el 13 de enero de 1657, en tanto el día 30 del mismo mes se ratifica ante el Teniente Mayor de Minas, Pablo Bernárdez de Ovando.

Al legislar sobre la manifestación de minas, Toledo advierte que es necesario tener especial consideración con los indígenas, a causa de su “incapacidad" (Lorente, [1573] 1867: 278), remitiéndonos a las apuntadas características que forjarían su condición. Las palabras que cierran la declaración de Vilca apuntan en esa dirección al autodefinirse como un "yndio yndefenso"5. Probablemente, y con independencia de quién fuera el autor de la petición, tras las expresiones de Vilca, quien parece conocer al dedillo las Ordenanzas toledanas que cita textualmente, se encuentre la voz de Cabrera Girón, beneficiario último del descubrimiento. Este vínculo se sustenta, con certeza, en la condición de baqueano del indígena quien evidentemente contaba con 
mayores recursos prácticos que el escribano a la hora de descubrir una veta de mineral. Pero no debemos descartar la ventaja que suponía su prerrogativa jurídica: la posibilidad de ahorrar tiempo haciendo la manifestación frente al Alcalde indígena, primer peldaño de la justicia en la jurisdicción.

\section{Conclusiones}

Entre la voluntad de imponer una cultura jurídica por parte de los conquistadores hasta la instancia en que un indígena se acerca al tribunal para pedir justicia, existe un vasto y desigual proceso de contactos y acercamientos. Para pensar este largo camino de consolidación de la justicia como una herramienta por parte de los indígenas, debemos rescatar la acción de los múltiples agentes que mediaron en ese proceso. Lenguaraces, jueces comisionarios, escribanos, además de protectores de naturales, alcaldes, caciques y otras autoridades étnicas y eclesiásticas serán los responsables de trasmitir y hacer asequible a los indígenas todo un corpus que, originalmente, les era ajeno (Jurado, 2010; Presta, 2013; Puente Luna y Honores, 2016; Cunill, 2019, entre otros). No debemos descuidar, claro está, la variable temporal. Probablemente, Diego Viltipoco represente, en este sentido, un ejemplo excepcional por la claridad con la que se maneja entre los diferentes ámbitos jurisdiccionales trazados por los conquistadores en un tiempo tan temprano.

Los casos presentados tienen como común denominador a la Real Audiencia de Charcas apareciendo como la autoridad de referencia. Si bien no podemos efectuar generalizaciones a partir de ello ya que el otorgamiento de potestades jurisdiccionales admite la prevalencia de dictámenes promulgados por ordenamientos inferiores sobre esferas de mayor alcance (Vallejos, 2009), sí es posible, en cambio, plantear el abanico de perspectivas disponibles que el orden impuesto admite y que los indígenas reconocen y usufructúan. A partir de ello, se establece una serie de trayectos que, de alguna manera, marcan una topografía de la justicia interétnica. Reconocer esta variable nos permitirá ahondar en el estudio de la justicia y pensar, además, la calidad del vínculo que se teje entre Charcas y los diferentes ámbitos de su jurisdicción.

Desde hace algunos años, se ha planteado a la distancia como una variable indispensable para abordar el ejercicio de la justicia (Barriera, 2012; 2013). En términos materiales, la dilatada y accidentada geografía del continente constituía un evidente obstáculo: el "gobierno de las provincias americanas instalará para la monarquía la cuestión de la extensión física como una verdadera variable jurídica" (Barriera, 2013: 144). Esto podía accionar tanto en la duración del proceso como en las posibilidades concretas de acceso a la justicia. Probablemente para los indígenas, reducidos y presuntamente controlados en sus pueblos, precisando de lenguaraces y otros intermediarios, estas distancias se extendiesen en un proceso donde la percepción difería de lo estrictamente geográfico.

De hecho, los tres casos presentados dan cuenta de indígenas que acceden a la Audiencia en condiciones particulares. Pensemos en Diego Viltipoco que al mando de indígenas rebeldes controlaba el 
camino que iba, justamente, entre la Gobernación y la Audiencia. Su figura era la que determinaba en buena medida la percepción de la distancia que medaba entre Charcas y Tucumán. En circunstancias muy diferentes, tanto Pedro Socomba como Francisco Vilca poseían un vínculo de cercanía con el corpus cultural del conquistador. Socomba como Cacique de Humahuaca tenía una historia compartida con las autoridades españolas en el proceso de evangelización de sus indígenas y en la administración de la economía regional, además del apoyo que suponía el Protector de Naturales para acceder a la Audiencia. Por su parte, su pericia como cateador en un ámbito de tanto interés como el minero allanaron el camino de Francisco Vilca que, no obstante, ejerció sus derechos en el ámbito de justicia más próximo.

Por último, nos interesa remarcar la importancia del acto de salvar distancias. Como marcamos en el caso de Socomba, el viaje que realiza en su rol de curaca hasta San Salvador de Jujuy debe interpretarse como un acto político que, en definitiva, posiciona la identidad de su propio pueblo (Rodríguez, 2011). También el traslado de las autoridades por el espacio debe verse en este sentido. En el caso de los oidores se dará a través de las visitas, una forma de teatro público que visibiliza el proceso de subordinación del pueblo de indios a la ciudad y remarca la colonialidad y el lugar menospreciado de los tributarios (Guevara Gil y Salomon, 1996). Antonio Hespanha (1989; 1993) entendía la necesidad de comprender las relaciones de poder a partir de las representaciones del espacio, lo inmaterial, así como los vínculos que forjaban el don de la gracia o las prácticas consuetudinarias. Consideramos que revisar los mapas jurisdiccionales a partir del uso que, en este caso, los indígenas hacen de las diversas instancias y comprender la función social que este aprovechamiento implica nos acerca a comprender la subjetividad del vínculo colonial y a profundizar nuestra mirada de la conformación del espacio surandino.

\section{Referencias citadas}

Agüero, A. (2013): “Derecho local y localización del derecho en la tradición jurídica hispana. Reflexiones a partir del caso de Córdoba del Tucumán”, En El derecho local en la periferia de la monarquía hispana: Río de la Plata, Tucumán y Cuyo. Siglos XVI-XVIII, pp. 91-120.

Agüero, A. (2012): “Expansión y colonización”, en Lorente M. y J. Vallejos (eds.), Manual de Historia del Derecho. Madrid: Tirant lo Blanch, pp. 225-258.

Agüero, A. (2008): Castigar y perdonar cuando conviene a la república. La justicia penal de Córdoba del Tucumán, siglos XVII y XVIII. Madrid: Centro de Estudios Políticos y Constitucionales.

Agüero, A. (2007): “Categorías básicas de la cultura jurisdiccional”, en Lorente, M. (coord.), De justicia de jueces a justicia de leyes. Hacia la España de 1870. Madrid. Consejo General del Poder Judicial, pp. 20-58.

Albeck M. E. y S. Palomeque (2009): “Ocupación española de las tierras indígenas de la puna y "raya del Tucumán" durante el temprano período colonial", en Memoria Americana 17 (2), pp. 173-212.

Angeli, S. (2016): "El ama, la justicia y el derecho: aportes sobre ley, derecho y altos tribunales de justicia en 
Hispanoamérica (siglos XVI- XVII)” en Res Gesta 52.

Barriera, D. (2012): “Instituciones, justicias de proximidad y derecho local en un contexto reformista: designación y regulación de 'jueces de campo' en Santa $\mathrm{Fe}$ (Gobernación-Intendencia de Buenos Aires) a fines del siglo XVIII", en Revista Historia del Derecho núm. 44. Buenos Aires.

Barriera, D. (2013): "Entre el retrato jurídico y la experiencia en el territorio", en Caravelle 4, 101 (dec.).

Becerra, M. F. (2014): “Para labrar y poblar”... Prácticas minero-metalúrgicas en la Puna de Jujuy durante el período colonial (siglos XVII- XVIII), Tesis doctoral inédita, Universidad de Buenos Aires, Buenos Aires.

Becerra, M. F. (2012): “Cruces entre Arqueología e Historia: las prácticas minero metalúrgicas coloniales en la Puna de Jujuy a través del complejo Fundiciones 1 (Rinconada, Jujuy, Argentina)", Población \& Sociedad, 19 (1), pp. 5-39.

Becerra, M. F. y D. Estruch (2011): "Alcaldes de minas, capitulares, cateadores y mineros. Una reflexión sobre las administración de la justicia en las causas mineras de la Puna de Jujuy (Siglos XVII y XVIII)", en Revista Historia del Derecho 42, Buenos Aires.

Clavero, B. (1994): Derecho indígena y cultura constitucional en América. Madrid, Siglo XXI.

Costa, P. (1969): Iurisdictio. Semantica del potere político medioevale (1100-1433). Milano, Giuffré.

Cunill, C. (2019): “Tribunales itinerantes, justicia localy mediación lingüística en Yucatán (siglo XVI)" en Cunill, C. y Luis Miguel Glave Testino (coords.), Las lenguas indígenas en los tribunales de América Latina: intérpretes, mediación y justicia (siglos XVI-XXI). Bogotá: Instituto Nacional Colombiano de Antropología e Historia, pp. 41-64.

Duve, T. (2005): “Los privilegios de los indios ¿Derecho local?", en Torres Aguilar M. (coord.), Actas del XV Congreso del Instituto Internacional de Historia del Derecho Indiano, Diputación de Córdoba, Universidad de Córdoba.

Estruch, D. (2012): “"Entre la Potestas y la Auctoritas'. Un recorrido histórico por el dualismo del poder en el occidente cristiano. Cultura y Religión”, Revista de Sociedades en Transición, VI (2), pp.78-102.

Estruch, D. (2009): "Fundar, gobernar y rezar. Una aproximación a los vínculos entre sociedad, política y religión en el Jujuy colonial (1656-1776)", Runa, XXX (1), pp. 61-78.

Garriga, C. (2010): “Concepción y aparatos de la justicia. Las Reales Audiencias de las Indias”, en Cuadernos de Historia, $\mathrm{N}^{\circ} 19$, Academia Nacional de Derecho y Ciencias Sociales de Córdoba, Instituto de Historia del Derecho y de las Ideas Políticas “Roberto I. Peña”, pp. 203-244.

Guevara Gil, A. y F. Salomón (1996): “Tradiciones culturales y transformaciones coloniales. Una 'visita personal de indios': ritual político en la Colonia y construcción del “indio" en los Andes”, en Cuadernos de Investigación 1, pp. 6-48. Instituto Riva- Agüero, Pontificia Universidad Católica del Perú, Lima.

Hespanha, A. (1989): Vísperas de Leviatán. Instituciones 
y poder político, Portugal, siglo XVII. Madrid: Taurus Humanidades.

Hespanha, A. (1993): La gracia del Derecho. Economía de la cultura en la Edad Moderna. Madrid: Centro de Estudios Constitucionales.

Hespanha, A. (2002): Culturajurídica europea. Síntesis de un milenio. Madrid: Tecnos.

Jurado C. (2010): “Don Pedro Dueñas, indio lengua. Un estudio de caso de la interpretación lingüística andino-colonial en el siglo XVII", en Anuario del Archivo y Biblioteca Nacional de Bolivia, núm. 16, pp. 285-309.

Levaggi, A. (2012): "Consideraciones sobre investigación en Historia del Derecho”, en IUSHistoria, núm. 5, USAL.

Levene, R. (1951): Las Indias no eran colonias. Madrid: Espasa Calpe.

Levillier, R. (1919-1920): Gobernación del Tucumán: probanza de méritos y servicios de los conquistadores. Buenos Aires, Colección de Publicaciones Históricas del Congreso Argentino.

Lizárraga R. ([1605] 1999): Descripción del Perú, Tucumán, Río de la Plata y Chile. Buenos Aires: Union académique Internationale. Academia Nacional de la Historia.

Matienzo, J. de ([1567] 1967): Gobierno del Perú, en Lohmann Villena, G. (ed.), París-Lima: Travaux de l'IFEA.

Lorente, S. ([1573] 1867): Relaciones de los Virreyes y Audiencias que han gobernado el Perú, Memorial y Ordenanzas de D. Francisco de Toledo. Lima: Imprenta del Estado por J. E. del Campo.

Nielsen, A. (2010): Celebrando los antepasados. Arqueología del espacio público en Los Amarillos. Quebrada de Humahuaca, Jujuy, Argentina. Buenos Aires: Mallku ediciones.

Oyarzábal, M. C. (2019): Ordenar una sociedad mestiza. Justicia, religión y costumbre, Jujuy, siglo XVII, Tesis doctoral inédita, Universidad Nacional de La Plata, La Plata.

Poloni Simard, J. (2005): “Los indios ante la justicia. El pleito como parte de la consolidación de la sociedad colonial”, en Lavallé B. (comp.), Máscaras, tretas y rodeos del discurso colonial en los Andes. Lima: IFEA/ PUCP/Instituto Riva Agüero, pp. 177-188.

Presta, A. M. (2013): "Redes de tinta y poder. Escribanos, clero e indígenas en la ciudad de La Plata", en Anuario del Archivo y Biblioteca Nacional de Bolivia, 18, Sucre, pp. 351-372.

Puente Luna, J. C. de la y R. Honores (2016): “Guardianes de la real justicia: alcaldes de indios, costumbre y justicia local en Huarochirí colonial”. Histórica XL (2), pp. 11-47.

Rodríguez, L. (2011): “El viaje de don Lorenzo y otros 'peregrinajes'. Reclamos territoriales, identidad y memoria en la comunidad de Amaicha del Valle", en Rodríguez, L. (comp.), Resistencias, conflictos y negociaciones. El valle Calchaquí desde el período prehispánico hasta la actualidad, Rosario: Prohistoria, pp. 123-144. 
Salas, M. (1945): El Antigal de Ciénaga Grande, Buenos Aires, Publicaciones del museo etnográfico de la facultad de Filosofía y Letras, Imprenta de la Universidad.

Sica, G. (2006): Del Pukara al Pueblo de Indios. El proceso de construcción de la sociedad indígena colonial en Jujuy, Argentina, siglo XVII, Tesis doctoral inédita, Universidad de Sevilla, Sevilla.

Sica, G. (2014a): “En torno al Corregidor de Omaguaca. Atribuciones, competencias y disputas jurisdiccionales en el Tucumán colonial, s. XVII", en Sierra, M. J. Pro y D.

Manero (comps.), Desde la Historia. Homenaje a Marta Bonaudo. Buenos Aires: Imago Mundi, pp. 108-208.

Sica, G. (2014b): "Las otras mitas. Aproximaciones al estudios de la mita de plaza en la jurisdicción de Jujuy, gobernación del Tucumán, sigloXVII", en Anuario de Estudios Americanos, 71 (1), pp. 201-226.

Solórzano Pereira J. De ([1648] 1703): Política indiana. Amberes: Henrico y Cornelio Verdussen.

Tau Anzoátegui, V. (1992): Casuismo y sistema. Indagación histórica sobre el espíritu del Derecho Indiano, Buenos Aires: Instituto de Investigaciones de Historia del Derecho.

Tau Anzoátegui, V. y E. Martiré (1996): Manual de Historia de las Instituciones argentinas. Buenos Aires: Macchi.

Tau Anzoátegui, V. y A. Agüero (coords.) (2013): El derecho local en la periferia de la Monarquía Hispana. Río de la Plata, Tucumán y Cuyo, siglos XVI- XVIII,
Buenos Aires: Instituto de Investigaciones de Historia del Derecho

Vallejos, J. (2009): “El cáliz de plata. Articulación de órdenes jurídicos en la jurisprudencia del Ius Commune", en Revista de Hisotria del Derecho, 38, pp. 1-13.

Zanolli, C. (2008): Entre la coerción, la oportunidad y la salvación. Las cofradías de indios de San Antonio de Humahuaca. Siglos XVII y XVIII, Salta: Andes.

Zanolli, C. (2005): Tierra, encomienda e identidad omaguaca. 1540-1638, Buenos Aires, Sociedad Argentina de Antropología.

\section{Notas}

${ }^{1} \mathrm{ATJ} 280,42 \mathrm{v}$

${ }^{2} \mathrm{ATJ} 280,44 \mathrm{r}$

${ }^{3}$ ABNB Min 62-4

${ }^{4}$ ABNB Min 62-4

${ }^{5} \mathrm{ABNB}$ Min $62-47 \mathrm{v}$ 